\title{
Biodistribution and pharmacokinetics of a telodendrimer micellar paclitaxel nanoformulation in a mouse xenograft model of ovarian cancer
}

This article was published in the following Dove Press journal:

International Journal of Nanomedicine

23 March 2012

Number of times this article has been viewed

\author{
Wenwu Xiao' \\ Juntao Luo ${ }^{2}$ \\ Teesta Jain ${ }^{3}$ \\ John W Riggs ${ }^{3}$ \\ Harry P Tseng' \\ Paul T Henderson ${ }^{3}$ \\ Simon R Cherry ${ }^{4}$ \\ Douglas Rowland ${ }^{4}$ \\ Kit S Lam ${ }^{1,3}$ \\ 'Department of Biochemistry and \\ Molecular Medicine, UC Davis Cancer \\ Center, University of California Davis, \\ Sacramento, CA; ${ }^{2}$ Department of \\ Pharmacology, SUNY Upstate Cancer \\ Research Institute, SUNY Upstate \\ Medical University, Syracuse, NY; \\ ${ }^{3}$ Department of Internal Medicine, \\ Division of Hematology and \\ Oncology, ${ }^{4}$ Department of Biomedical \\ Engineering, UC Davis Cancer Center, \\ University of California Davis, \\ Davis, CA
}

Correspondence: Kit Lam

Department of Biochemistry and

Molecular Medicine, UC Davis Cancer

Center, University of California Davis,

Sacramento, CA 95817, USA

$\mathrm{Tel}+\mathrm{I} 9167340910$

Fax + I9167346415

Email kit.lam@ucdmc.ucdavis.edu

Correspondence: Juntao Luo

Department of Pharmacology, SUNY

Upstate Cancer Research Institute, SUNY

Upstate Medical University, 750 East

Adams Street, Syracuse, NY 13210 , USA

Tel +I 3154647965

$\mathrm{Fax}+|3| 54645143$

Email luoj@upstate.edu
Background: A multifunctional telodendrimer-based micelle system was characterized for delivery of imaging and chemotherapy agents to mouse tumor xenografts. Previous optical imaging studies demonstrated qualitatively that these classes of nanoparticles, called nanomicelles, preferentially accumulate at tumor sites in mice. The research reported herein describes the detailed quantitative imaging and biodistribution profiling of nanomicelles loaded with a cargo of paclitaxel.

Methods: The telodendrimer was covalently labeled with ${ }^{125} \mathrm{I}$ and the nanomicelles were loaded with ${ }^{14} \mathrm{C}$-paclitaxel, which allowed measurement of pharmacokinetics and biodistribution in the mice using microSPECT/CT imaging and liquid scintillation counting, respectively.

Results: The radio imaging data showed preferential accumulation of nanomicelles at the tumor site along with a slower clearance rate than paclitaxel formulated in Cremophor EL $\left(\operatorname{Taxol}^{\circledR}\right)$. Liquid scintillation counting confirmed that ${ }^{14} \mathrm{C}$-labeled paclitaxel sequestered in nanomicelles had increased uptake by tumor tissue and slower pharmacokinetics than Taxol.

Conclusion: Overall, the results indicate that nanomicelle-formulated paclitaxel is a potentially superior formulation compared with Taxol in terms of water solubility, pharmacokinetics, and tumor accumulation, and may be clinically useful for both tumor imaging and improved chemotherapy applications.

Keywords: telodendrimer, nanomicelle, paclitaxel, microSPECT/CT, imaging guided drug delivery

\section{Introduction}

Cancer is primarily characterized by the uncontrolled proliferation of cells and their ability to metastasize. In spite of significant advances in the fight against cancer, it remains a challenging medical problem. Systemic chemotherapy is a common approach to the treatment of cancer. However, toxicity is a major side effect, which limits the utility and effectiveness of such nontargeted chemotherapeutics. Recent research in the development of drug delivery systems has focused on targeted delivery and controlled drug release at tumor sites in order to increase efficacy while reducing systemic toxicity. Imaging-guided drug delivery is considered a promising strategy to achieve this goal. ${ }^{1}$ Full implementation of imaging-guided drug delivery will require that drugs can be imaged or identified in the body as they enter the blood stream. The goal of imaging-guided drug delivery is to optimize local delivery of the therapeutic pharmaceutical agent to the target tissue and provide microanatomical and functional imaging feedback of the therapeutic process, as well as longitudinal treatment and monitoring. 
The explosive growth of biocompatible nanotechnologies has made the clinical utilization of molecular imaging and therapy with a host of novel agents a realistic near-term possibility. Various nanocarriers, such as superparamagnetic iron oxide nanoparticles, ${ }^{2,3}$ gold nanoparticles, ${ }^{4}$ liposomes,${ }^{5}$ and polymeric nanoparticles ${ }^{6}$ loaded with large payloads of imaging agents, enable detection of cancer with standard imaging equipment via passive or active tumor-targeting effects. In order to fulfill the goal of imaging-guided drug delivery, nanocarriers need to be multifunctional, thus making them useful for both imaging and drug delivery. Our group has developed a well-defined and biocompatible amphiphilic telodendrimer system composed of polyethylene glycol (PEG), cholic acid, and a dendritic lysine core that can self-assemble into multifunctional water-soluble nanomicelles to enable efficient delivery of hydrophobic cargos such as paclitaxel and the hydrophobic dye, 1, 1'-dioctadecyl$3,3,3^{\prime}, 3^{\prime}$-tetramethylindodicarbocyanine perchlorate. ${ }^{7,8}$ Telodendrimers are easily labeled with fluorophores and radionuclides by covalent conjugation onto a lysine side chain at the junction site between the PEG and the cholic acid cluster, without compromising their propensity to form nanomicelles. For noncovalent labeling, telodendrimers can be mixed with radio-labeled drugs or fluorescent probes during nanomicelle formation. ${ }^{7}$ The tailored multifunctional features of the telodendrimer-based nanomicelle system are ideal for imaging-guided drug delivery applications.

Previous reports ${ }^{7,8}$ have demonstrated efficient tumor targeting and drug delivery by nanomicelles through optical imaging and efficacy studies using ovarian cancer xenograft models. Our nanomicelles were prepared via self-assembly of telodendrimers containing a linear $5 \mathrm{kDa}$ PEG covalently attached to a dendritic lysine core with eight cholic acid groups $\left(\mathrm{PEG}^{5 \mathrm{k}} \mathrm{CA}_{8}\right)$. In order to characterize and quantitatively analyze the biodistribution and tumor targeting effect of nanomicelle nanocarriers further, the telodendrimer was radiolabeled with ${ }^{125} \mathrm{I}$ using the Bolton-Hunter reagent, which allowed in vivo microSPECT/CT imaging studies. In separate experiments, ${ }^{14} \mathrm{C}$-labeled paclitaxel $\left({ }^{14} \mathrm{C}\right.$-PTX $)$ was loaded into the nanomicelles $\left({ }^{14} \mathrm{C}-\mathrm{PTX}-\mathrm{NM}\right)$ and administered to mice bearing tumors in order to elucidate the tumor-targeting, biodistribution, and pharmacokinetic profiles of the drug cargo sequestered in the nanocarrier. Comparison of both the imaging and quantitative biodistribution profiles of paclitaxel loaded into nanomicelles will aid in understanding and optimizing the telodendrimer system for better targeting of systemic cancer therapies.

\section{Materials and methods}

\section{Paclitaxel}

${ }^{14} \mathrm{C}-\mathrm{PTX}$ was purchased from Moravek Biochemicals Inc, (Brea, CA). Unlabeled paclitaxel was purchased from AK Scientific (Union City, CA). A Cremophor EL formulation of paclitaxel $\left(\right.$ Taxol $^{\mathbb{}}$ ) from AK Scientific (Mountain View, CA) was obtained from the UC Davis Cancer Center pharmacy.

\section{Cell lines}

Ovarian cancer (SKOV-3) cell line was obtained from the American Type Culture Collection (Manassas, VA). The cells were maintained in Macoy-5A media and split every 3-4 days.

\section{Mouse ovarian cancer xenograft model}

The animal studies were performed according to a protocol approved by the Institutional Animal Care and Use Committee of the University of California, Davis. Female athymic nude mice (nu/nu), obtained from Harlan Inc, (Indianapolis, IN) at 5-6 weeks of age, were injected subcutaneously in the right flank with $5 \times 10^{6} \mathrm{SKOV}-3$ cells suspended in $200 \mu \mathrm{L}$ of phosphate-buffered saline. When the subcutaneous tumors reached $0.5-1.0 \mathrm{~cm}$ in diameter or 14-21 days after implantation, the tumor-bearing mice were subjected to microSPECT/CT imaging of the ${ }^{125} \mathrm{I}$-labeled nanomicelles or biodistribution studies of the ${ }^{14} \mathrm{C}-\mathrm{PTX}$ loaded nanomicelles.

\section{lodination of telodendrimers and micelles}

Iodination of telodendrimers was accomplished via the ${ }^{125}$ I-Bolton-Hunter reagent, which was provided by Perkins Elmer through custom radiolabeling. Excess Bolton-Hunter reagent was removed by passage through an LH-20 size exclusive column using ethanol as the eluent, yielding $10 \mathrm{mCi}$ of ${ }^{125} \mathrm{I}$-labeled telodendrimers with a radioactive purity of $99.1 \%$.

Preparation of the paclitaxel-loaded ${ }^{125}$ I-labeled nanomicelles (PTX-125I-NM) was as follows: $3.2 \mathrm{mg}$ of paclitaxel and $32 \mathrm{mg}$ of $\mathrm{PEG}^{5 \mathrm{k}} \mathrm{CA}_{8}$ telodendrimer were dissolved in $2 \mathrm{~mL}$ of chloroform. Immediately after mixing, $0.8 \mathrm{mCi}$ of ${ }^{125} \mathrm{I}$-labeled $\mathrm{PEG}^{5 \mathrm{k}} \mathrm{CA}_{8}$ solution in ethanol $(0.32 \mathrm{~mL})$ was added. The solution was dried via nitrogen blowing overnight in a radio safety fume hood. Phosphate-buffered saline $1.6 \mathrm{~mL}$ was added and the mixture was vortexed for 30 seconds and sonicated for 30 minutes to disperse the complex into micelles. The ratio of ${ }^{125}$ I-labeled to unlabeled $\mathrm{PEG}^{5 \mathrm{k}} \mathrm{CA}_{8}$ in the paclitaxel-loaded nanomicelles was $1: 800$ by mass. 


\section{MicroSPECT/CT imaging}

MicroSPECT/CT imaging of PTX- ${ }^{125} \mathrm{I}-\mathrm{NM}$ in a human ovarian cancer xenograft model was performed at the Center for Molecular and Genomic Imaging, University of California, Davis. Nude mice bearing SKOV-3 xenografts were injected with $200 \mu \mathrm{L}$ of $100 \mu \mathrm{Ci}$ paclitaxel-loaded PTX- ${ }^{125}$ I-NM (400 $\mu \mathrm{g}$ of paclitaxel in $4 \mathrm{mg}$ of $\mathrm{PEG}^{5 \mathrm{k}} \mathrm{CA}_{8}$ ) via the tail vein. Pinhole SPECT images were acquired at hours 1, 5, 18, 24, 48, 72, and 94 following injection. SPECT images were acquired using the InveonMM (Siemens Preclinical Solutions, Knoxville, TN). The collimator set used was the mouse medium energy collimator, which is a single pinhole with a $3 \mathrm{~mm}$ diameter. The scanner has two SPECT heads that rotate around the animal. The radius of rotation was set at $40 \mathrm{~mm}$. This collimator and radius combination yields a spatial resolution of approximately $3.5 \mathrm{~mm}$. A total of 20 projections were acquired over 180 degrees per SPECT head and the initial projection exposure time was 150 seconds. The exposure time at each projection is adjusted for nuclide decay to yield an equivalent number of counts per projection. List mode data are collected, including all photon events, and photon energy is recorded. The data were reconstructed with an energy cut of 28-42 keV. The images were reconstructed with OSEM using four subsets and 20 iterations. Low resolution CT images were acquired for anatomical registration with the SPECT images. The images are displayed as a percent of the maximum voxel in the image. All images were scaled to 100 as the maximum intensity value.

\section{${ }^{14} \mathrm{C}-\mathrm{PTX}-\mathrm{NM}$ preparation}

The micelles were formulated in a manner similar to the protocol used above, except without ${ }^{125}$ I-labeling. Briefly, $4 \mu \mathrm{Ci}$ of ${ }^{14} \mathrm{C}$-PTX in ethyl acetate was added to $5 \mathrm{~mL}$ of chloroform solution containing $15 \mathrm{mg}$ of unlabeled paclitaxel and $100 \mathrm{mg}$ of $\mathrm{PEG}^{\mathrm{sk}} \mathrm{CA}_{8}$ telodendrimer. The solution was dried by nitrogen blowing overnight, followed by addition of $5 \mathrm{~mL}$ of phosphate-buffered saline and vortex mixing for 30 seconds and sonication for 30 minutes. The ratio of radiocarbon-labeled and unlabeled paclitaxel in the resulting micelle formulation was approximately $1: 1100$ by mass.

Doping of Taxol with ${ }^{14} \mathrm{C}$-PTX $\left({ }^{14} \mathrm{C}\right.$-PTX-Taxol) was achieved as follows: $3 \mu \mathrm{Ci}$ of ${ }^{14} \mathrm{C}$-PTX in ethyl acetate was added to $1.875 \mathrm{~mL}$ of Taxol (6 mg paclitaxel $/ \mathrm{mL})$ and vortexed for 30 seconds. The Cremophor solution of Taxol was then diluted with phosphate-buffered saline to a final paclitaxel concentration of $1.5 \mathrm{mg} / \mathrm{mL}$. The ratio of radiocarbon-labeled and unlabeled paclitaxel in the resulting micelles was approximately 1:1100 by mass.

\section{${ }^{14} \mathrm{C}-\mathrm{PTX}$ biodistribution}

Nude mice bearing SKOV-3 xenografts were injected with $200 \mu \mathrm{L}$ solutions containing of $100 \mu \mathrm{Ci}{ }^{14} \mathrm{C}$-PTX-NM (400 $\mu \mathrm{g}$ total mass of paclitaxel that was mixed with $4 \mathrm{mg}$ of $\mathrm{PEG}^{5 \mathrm{k}} \mathrm{CA}_{8}$ prior to nanomicelle synthesis) via the tail vein. The major organs and tissues collected were whole blood, brain, skin, heart, lung, liver, spleen, kidney, bladder, muscle, and tumor. The time points of data collection were at hours $0,0.5,1,6,16,24,48$, and 72. At selected time points, blood was collected by heart puncture under temporary anesthesia with ether. After blood collection, the mouse was euthanized by cervical dislocation. All organs were collected and weighed, put into $1.5 \mathrm{~mL}$ Eppendorf centrifuge tubes, and kept on ice prior to homogenization. Organs and tissue were minced into chunks of approximately $1 \mathrm{~mm}$ in diameter. The minced tissue was then added to $1 \mathrm{~mL}$ of Biosol, agitated gently, and placed in an incubator shaker at $50^{\circ} \mathrm{C}$ and shaken for 6 hours at $650 \mathrm{rpm}$. When chunks of tissue were no longer visible in the suspension, all the solution in the tubes were transferred into $20 \mathrm{~mL}$ scintillation vials, and decolorized by addition of $0.2 \mathrm{~mL}$ of $30 \%$ hydrogen peroxide. The vials were capped loosely and incubated at $50^{\circ} \mathrm{C}$ for one hour. Finally, $18 \mathrm{~mL}$ of liquid scintillation counting cocktail was added into each scintillation vial, followed by running the samples in a Packard 1500 Tri-Carb liquid scintillation analyzer with standard protocols for radiocarbon detection. Data were processed on an Excel spreadsheet running Solutions pharmacokinetic software.

\section{Data processing and statistics}

For determination of the radiointensity of tumors and normal organs in the radioimaging, we calculated the average values of the tumor area and of the normal organ area using the three-dimensional regions of interest function of the Siemens microPET ASIPro WM 6.7.1.2 software program. All data are shown as the mean \pm standard deviation of three independent measurements. The Student's $t$-test was used for statistical analysis of the radioimaging intensity and pharmacokinetic data. Statistical significance was indicated by $P<0.05$ and $P<0.001$.

\section{Results and discussion MicroSPECT/CT imaging of nanomicelles}

Our previous research has demonstrated excellent tumor targeting of telodendrimer-based nanomicelles using near- 
infrared fluorescence imaging, whereby nanomicelles formed by $\mathrm{PEG}^{5 \mathrm{k}} \mathrm{CA}_{8}$ are loaded with the near-infrared dye, 1,1'-dioctadecyl-3,3,3',3'-tetramethyl indodicarbocyanine perchlorate, and paclitaxel., ${ }^{7,8}$ Near-infrared fluorescence imaging offers unique advantages for diagnostic imaging of solid tumors with high sensitivity, ${ }^{9}$ given the low in vivo autofluorescence in the near-infrared range (700-900 nm) and the minimal near-infrared absorbance by biological components, such as hemoglobin, water, and lipids. ${ }^{10,11}$ However, near-infrared fluorescence imaging is not quantitative and has a limited penetration distance, as well as low spatial resolution. ${ }^{12}$ In contrast, radioimaging is superior for quantification due to good tissue penetration of gamma rays and the ability to measure count rates in tissue accurately, permitting whole body quantitative imaging not only in small animals, but also in humans.

For this report, radioimaging was performed using an established tumor xenograft model. The $\mathrm{PEG}^{5 \mathrm{k}} \mathrm{CA}_{8}$ telodendrimer was labeled with ${ }^{125} \mathrm{I}$ via the Bolton-Hunter reagent as shown in Scheme 1A. The unbound labeling reagent was removed by passage through a size-exclusive column, with ethanol as the eluent to avoid formation of telodendrimer aggregates in which the iodination reagent could become trapped. The ${ }^{125} \mathrm{I}$-labeled telodendrimer was doped into a solution of unlabeled telodendrimers and loaded with paclitaxel during self-assembly according to a previously published protocol. ${ }^{7,8}$ Doses of PTX- ${ }^{125}$ I-labeled nanomicelles were injected into nude mice bearing SKOV-3 xenografts. As shown in Figure 1, decay-corrected imaging indicated that the PTX- ${ }^{125}$ I-NM accumulated specifically and continuously at the tumor site and reached a peak at around 18-24 hours post injection. The tumor signal then decreased gradually, with substantial retention of nanomicelles in the tumor with respect to background tissue for up to 94 hours post injection. Within the first 5 hours, the majority of the ${ }^{125} \mathrm{I}$-labeled material was observed in the blood circulation of the mouse and in the blood-rich organs of the chest and upper abdomen. The signal within normal organs, such as the heart, lung,

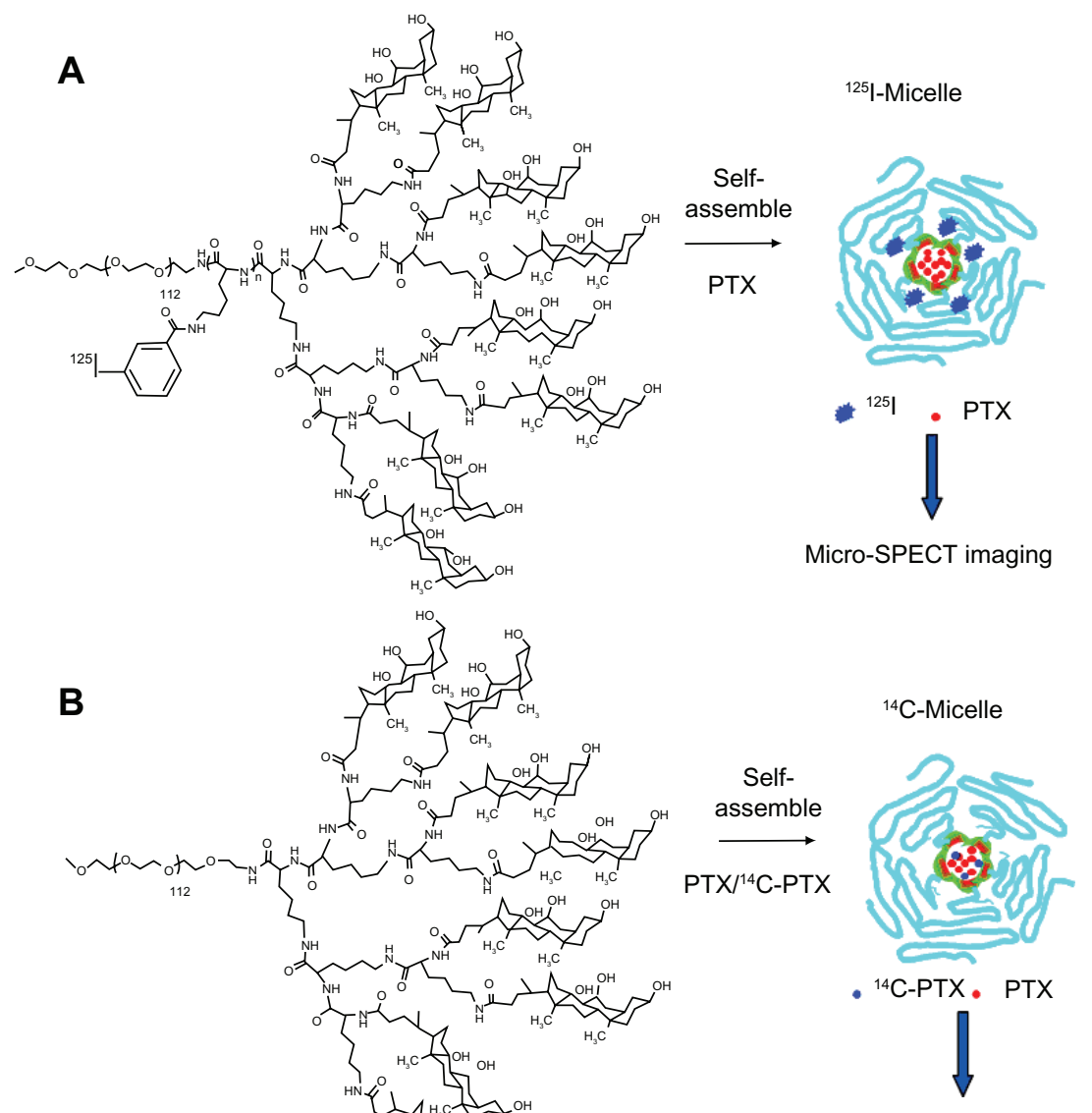

PK and biodistribution study

Scheme I Radioformulated nanomicelle. (A) Structure of the ${ }^{125}$-labeled telodendrimer PEG $^{5 k} C A_{8}$ and PTX-125I-NM for in vivo microSPECT imaging. (B) Structure of the parent telodendrimer $\mathrm{PEG}^{5 k} \mathrm{CA}_{8}$ and self-assembled micelles loaded with paclitaxel doped with ${ }^{14} \mathrm{C}$-PTX.

Abbreviations: ${ }^{14} \mathrm{C}-\mathrm{PTX},{ }^{14} \mathrm{C}$-labeled paclitaxel; NM, nanomicelles; PEG, polyethylene glycol; CA, cholic acid; $\mathrm{PEG}^{5 \mathrm{k}} \mathrm{CA}_{8}$, dendritic lysine core and eight cholic acid groups. 


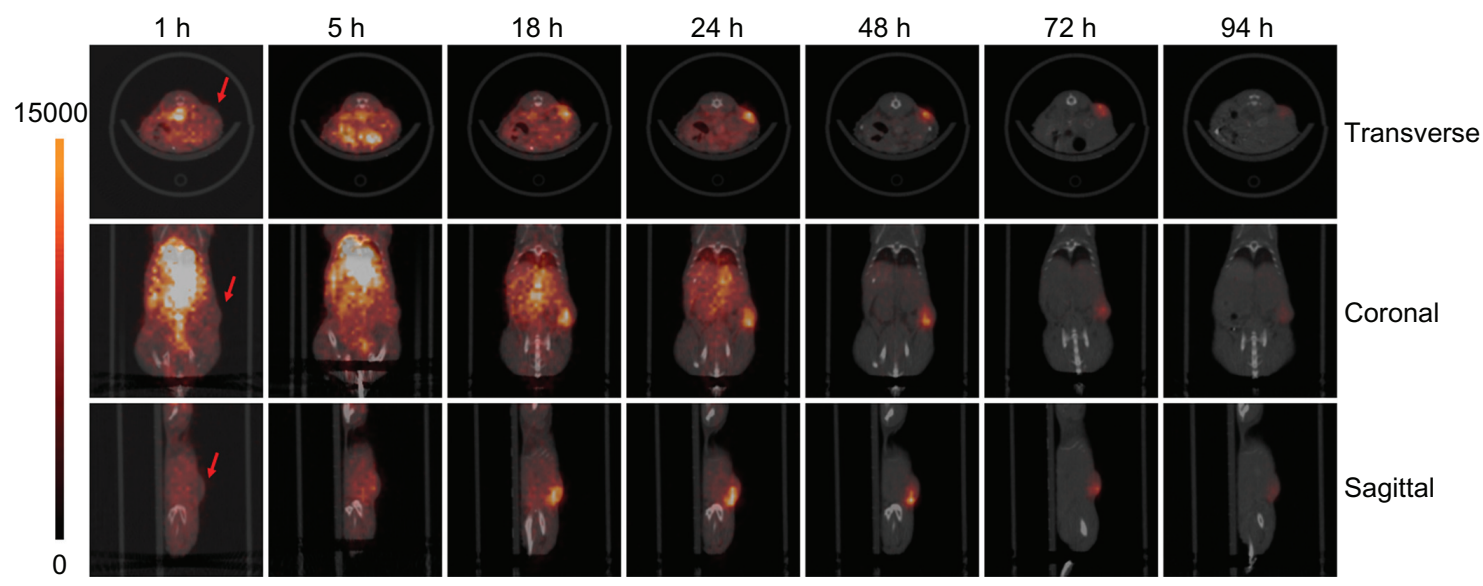

Figure I Decay-corrected microSPECT/CT imaging of nude mice bearing SKOV-3 ovarian cancer xenograft (red arrow) after PTX-125/-NM was injected through the tail vein. After injection of PTX-125/-NM, microSPECT/CT images were taken at hours I, 5, 18, 24, 48, 72, and 94. The panels show nanomicelle accumulation in tumor tissue with time, which reached its highest level at around 18-24 hours post injection, and then washed out gradually. The normal organs, including the heart, liver, and lung, demonstrated much higher uptake than tumor tissue within the first hour, but the signal decreased dramatically thereafter compared with that in tumor tissue.

Abbreviations: PTX, paclitaxel; NM, nanomicelles; PEG, polyethylene glycol; CA, cholic acid.

and liver, was dramatically higher than that in tumor tissue. For 18-24 hours post injection, the signal in the tumor tissue was significantly $(P<0.05)$ higher than in most of the rest of body. Forty-eight hours post injection, significant retention of PTX- ${ }^{125}$ I-NM persisted at the tumor site. Whole body projection images (Figure 2) showed the tumor to be the brightest part of the whole body at 72 hours post injection. In general, the tumor-targeting profile demonstrated by radioimaging is consistent with previous results from optical imaging, in which the telodendrimer was fluorescently labeled with BODIPY, a hydrophobic near-infrared dye. ${ }^{13}$ This finding supports previous observations that nanomicelles are able to target the tumor site via the enhanced permeability and retention effect associated with porous tumor vasculature, which enables nanomicelles to release their cargo into localized tumor regions.

Quantitative analysis of the imaging data was performed in order to study the distribution profiles of PTX- ${ }^{125} \mathrm{I}-\mathrm{NM}$ further in normal organs and tumor tissue. The radio signal in the blood pool refers to the signal calculated from the heart cavity. Other normal organs, such as the liver, lung, and muscle, were analyzed in addition to tumor tissue. Dominant ${ }^{125} \mathrm{I}$ accumulation was shown in the blood pool, liver, and lung within 24 hours. Distribution of ${ }^{125} \mathrm{I}$ in the liver was much higher than that in tumor tissue for 5 hours post injection (Figure $3 \mathrm{~A}$ ), which is probably due to the high content of blood in the liver. Early accumulation in normal tissues, including muscle, had washed out quickly by one hour post injection. However, uptake in tumor tissue increased gradually and reached a plateau at 18-24 hours, then decreased slowly. This contrast in accumulation between tumor and normal tissue is consistent with enhanced permeation and retention-mediated accumulation of nanomicelles in tumor tissue. The ${ }^{125} \mathrm{I}$ signal in normal organs and tumor over time was normalized by the signal in muscle to reflect the relative distribution and kinetic profiles. The signal change in muscle is usually in response to the basic clearance profile of the injected PTX- ${ }^{125}$ I-NM. Therefore, the signal in tumor tissue or organs normalized by the signal from muscle will subtract interference from the background to highlight accumulation

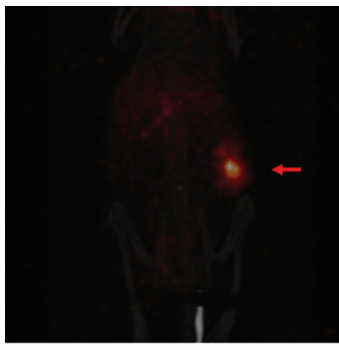

Back to front

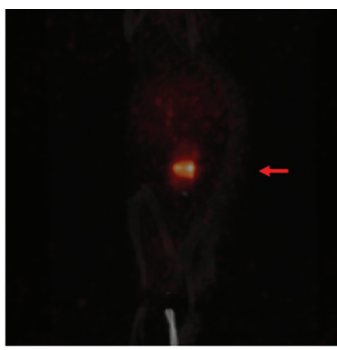

Left to right

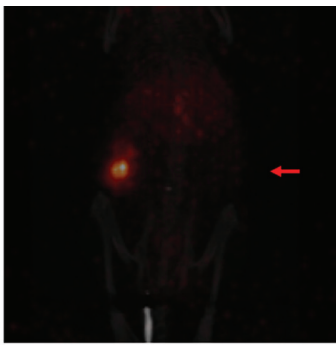

Front to back

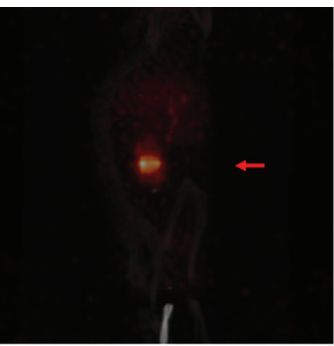

10000

Right to left

Figure 2 Projection microSPECT/CT imaging of nude mice bearing SKOV-3 xenografts (red arrow) 72 hours post injection. In four different projection positions, the ovarian cancer xenograft showed much greater uptake of nanomicelles in comparison with the rest of the body at 72 hours post injection. 


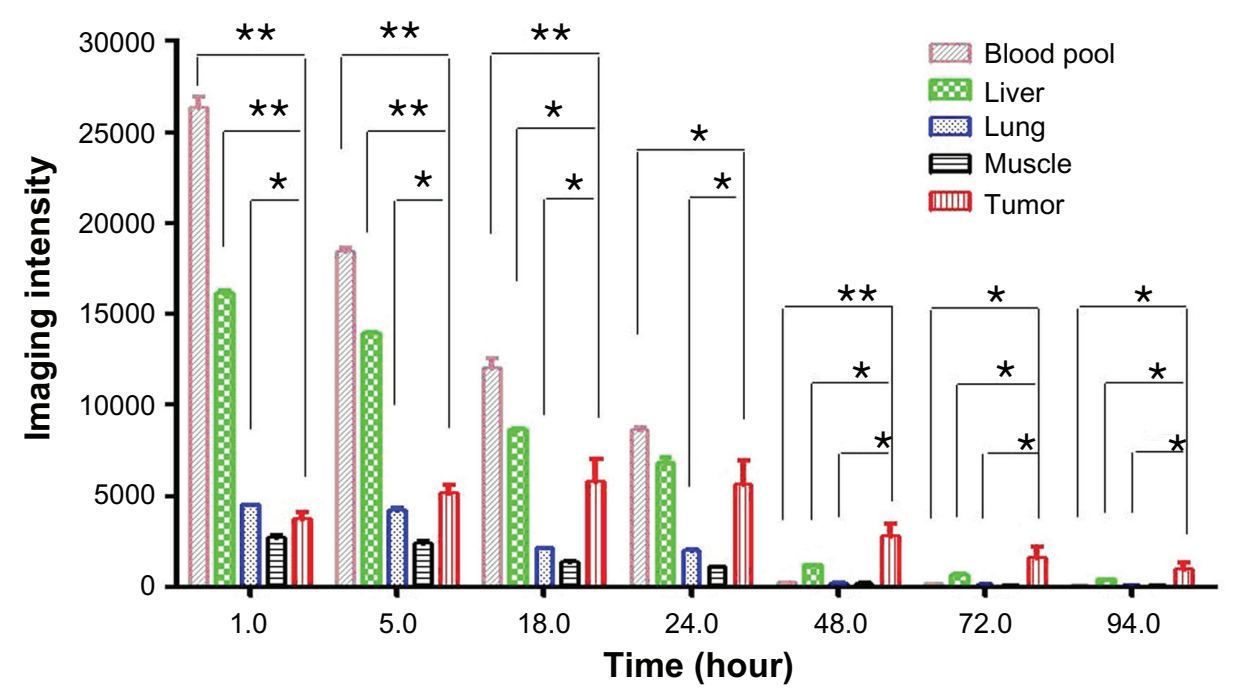

Figure 3A Distribution of PTX- ${ }^{125}$-NM in major organs and tumor tissue based on quantification of images. Within 24 hours, the radioactive signal in the blood pool was much higher than in the rest of the body and decreased rapidly to background levels by 48 hours. The liver and lung showed higher uptake of PTX-125I-NM than tumor tissue for one hour, but decreased very rapidly within 48 hours. However, radio signal accumulation in the tumor tissue increased to a peak within 24 hours, then decreased gradually thereafter.

Notes: $* P<0.05 ; * * P<0.01$.

Abbreviations: PTX, paclitaxel; NM, nanomicelles.

and clearance trends of PTX- ${ }^{125} \mathrm{I}-\mathrm{NM}$ in tumor tissue or organs. In addition, it will facilitate comparison between tumor tissue and normal organs. The normalized results indicate that the blood pool/muscle ratio was higher than the ratios from other organs and tumor tissue within 24 hours, but decreased rapidly to the background level by 48 hours. The ratio of lung/muscle remained constant, reflecting the similar clearance profile of the ${ }^{125}$ I-labeled substance in lung and muscle. However, the ratio of tumor/muscle kept increasing over time, indicating gradual accumulation and much slower clearance of the nanoparticles from tumor tissue. The liver/ muscle ratio slightly increased over time, probably owing to the reticuloendothelial system in the liver that continuously takes up the ${ }^{125}$ I-labeled materials remaining in the body (Figure 3B).

\section{Comparison of biodistribution of ${ }^{14} \mathrm{C}$-PTX-NM and ${ }^{14} \mathrm{C}$-PTX-Taxol}

An understanding of the fate and biological effects of nanoparticles in animals is critical to their medical applications

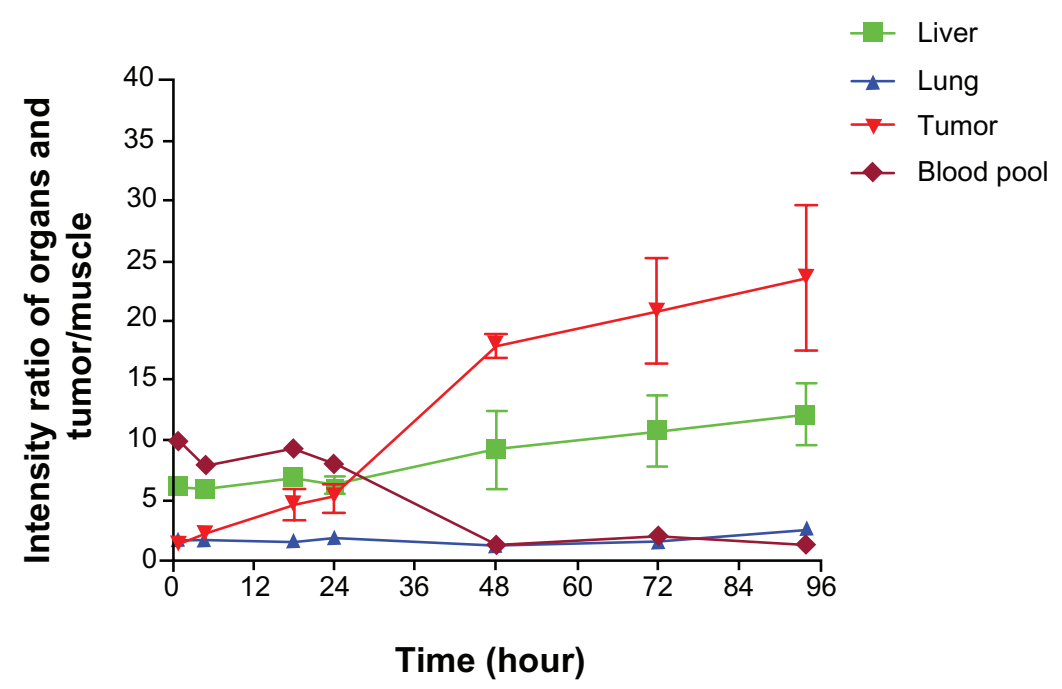

Figure 3B Imaging intensity ratio of normal organs and tumor tissue versus muscle.

The ratio of blood pool/muscle was highest shortly after injection, but kept decreasing dramatically to the background level of radioactivity within 48 hours. The ratio of lung/muscle did not increase noticeably. The tumor/muscle ratio increased much faster over 90 hours than the liver/muscle ratio, even though it was lower than that of liver/ muscle initially (within 24 hours). 
in vivo. Pharmacokinetic and organ/tissue distribution properties of nanoparticles are of great interest from the clinical point of view because of their potential uses in cancer imaging and therapy. Systematic studies are required to evaluate the distribution of administered nanoparticles inside the body, especially at the tumor site, after injection.

The use of ${ }^{14} \mathrm{C}$-PTX enabled us to quantify the drug and its metabolites within the mouse tissue and organs accurately via liquid scintillation counting after intravenous administration. Because the predominant metabolites of paclitaxel include 3'-p-hydroxypaclitaxel, $6 \alpha$-hydroxypaclitaxel, and $6 \alpha, 3^{\prime}$-p $p$-dihydroxypaclitaxel, without any metabolic loss of carbon atoms from the parent molecule, we assume that the radiocarbon measurements accounted for all of the parent paclitaxel and its metabolites present in each sample. ${ }^{14} \mathrm{In}$ order to study the effects of formulation into nanomicelles on the distribution of paclitaxel, ${ }^{14} \mathrm{C}$-PTX was loaded into nanomicelles and injected into nude mice bearing SKOV-3 xenografts. As a benchmark for comparison, a small amount of ${ }^{14} \mathrm{C}$-PTX was added to Taxol, a Cremophor EL formulation of paclitaxel used in the clinic, which was also administered to the tumor-bearing mice. The tissues and organs were harvested at various time points after injection. The ${ }^{14} \mathrm{C}$ concentration in blood and tissues was determined by liquid scintillation counting, which allowed calculation of total paclitaxel using the specific activity of each dose as a conversion factor. As shown in Figure 4, the biodistribution profiles of the ${ }^{14} \mathrm{C}$-PTX-NM in different organs and tumor tissue were similar to that of ${ }^{14} \mathrm{C}$-PTX-Taxol. Both formulations showed low brain uptake of paclitaxel, indicating a general lack of accessibility because of the blood-brain barrier. At the zero time point, blood was withdrawn by heart puncture within one minute of intravenous injection of the ${ }^{14} \mathrm{C}$-labeled drug formulation. Organs and tumor tissue were harvested immediately after euthanasia. Samples from all subsequent time points were harvested using the same procedure. Figure 4A shows that ${ }^{14} \mathrm{C}$ uptake in most of the organs reach a maximum within minutes, followed by rapid washout of drug, as was observed in the ${ }^{125} \mathrm{I}$ experiments. In contrast, the concentration of paclitaxel and its metabolites showed slower accumulation in tumor tissue, and reached a peak at around 30 minutes post injection, which then decreased slowly compared with normal tissues. The paclitaxel concentration in tumors of animals treated with the telodendrimer nanomicelle formulation was significantly $(P<0.05)$ higher than that in other organs, even in the liver, after 24 hours, which is consistent with the microSPECT/ $\mathrm{CT}$ imaging results. Tumor uptake in animals treated with ${ }^{14} \mathrm{C}$-PTX-Taxol was also observed to increase within the first hour and to decrease gradually to a level similar to that in other organs, including skin, lung, and liver (Figure 4B). It is known that Taxol, which is a Cremophor EL (surfactant)

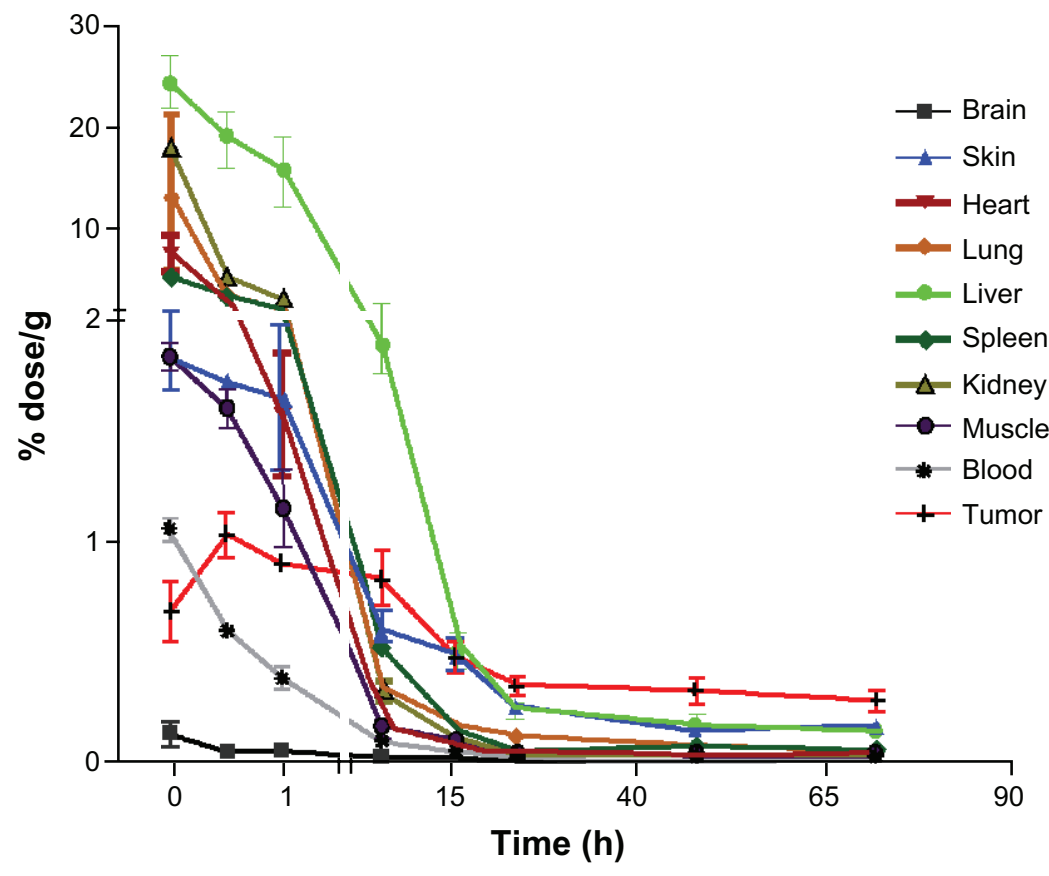

Figure 4A Biodistribution of ${ }^{14} \mathrm{C}$-labeled paclitaxel loaded into nanomicelles in nude mice with SKOV-3 xenografts. ${ }^{14} \mathrm{C}$-PTX-NM showed much higher uptake in normal organs, including the spleen, heart, lung, kidney, and in particular the liver, than in tumor tissue in the beginning, but decreased very rapidly within 16 hours. ${ }^{14} \mathrm{C}$-PTX-NM accumulation in tumor tissue reached a peak at 30 minutes post injection, then decreased gradually, and showed higher levels than that in all normal organs 16 hours post injection.

Abbreviations: ${ }^{14} \mathrm{C}$-PTX, ${ }^{14} \mathrm{C}$-labeled paclitaxel; NM, nanomicelles. 


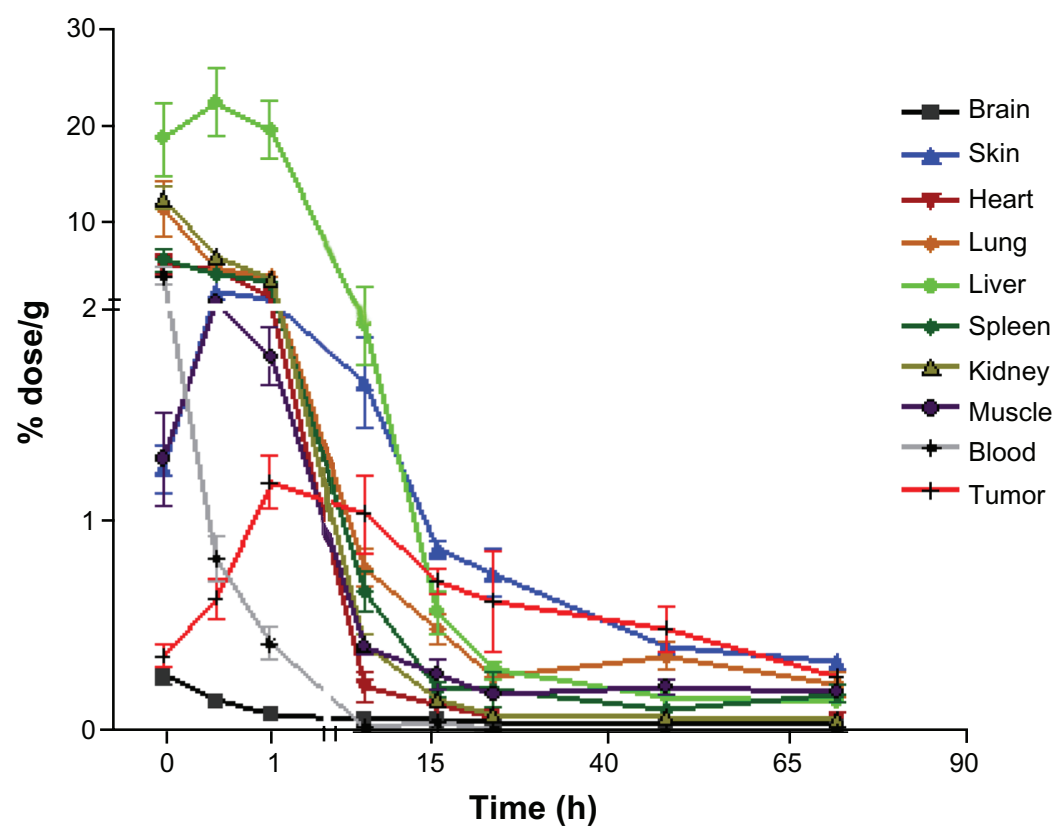

Figure 4B Biodistribution of ${ }^{14} \mathrm{C}$-labeled paclitaxel in $\mathrm{TaxO}^{\circledR}$ in nude mice with subcutaneous ovarian SKOV-3 xenografts.

${ }^{14} \mathrm{C}$-PTX (Taxol) accumulation in tumor tissue reached a peak at one hour post injection, then decreased gradually, showing levels close to those in normal organs 16 hours post injection. ${ }^{14} \mathrm{C}$-PTX showed much higher uptake in normal organs, including the heart, spleen, lung and kidney, especially in the liver, compared with tumor tissue in the beginning, but decreased very rapidly within 24 hours.

Abbreviation: ${ }^{14} \mathrm{C}$-PTX, ${ }^{14} \mathrm{C}$-labeled paclitaxel.

formulation of paclitaxel, can also form micelles of about $10 \mathrm{~nm}$ in size upon dilution in water or biological fluids. Therefore, Taxol also has a relatively long circulation time and is able to target tumor tissue via the enhanced permeation and retention effect. The biodistribution of Taxol in rodents has been described in tumor-bearing mice before. Liver and tumor have shown high paclitaxel concentrations at 24 hours after administration. ${ }^{15}$ Similar distribution behavior has also been observed with ${ }^{111}$ In-labeled paclitaxel. ${ }^{16}$ However, the stability of paclitaxel dissolved in Cremophor EL is poor in vitro upon dilution, as shown in our experiments. Paclitaxel has a tendency to leak out from the Cremophor micelles,

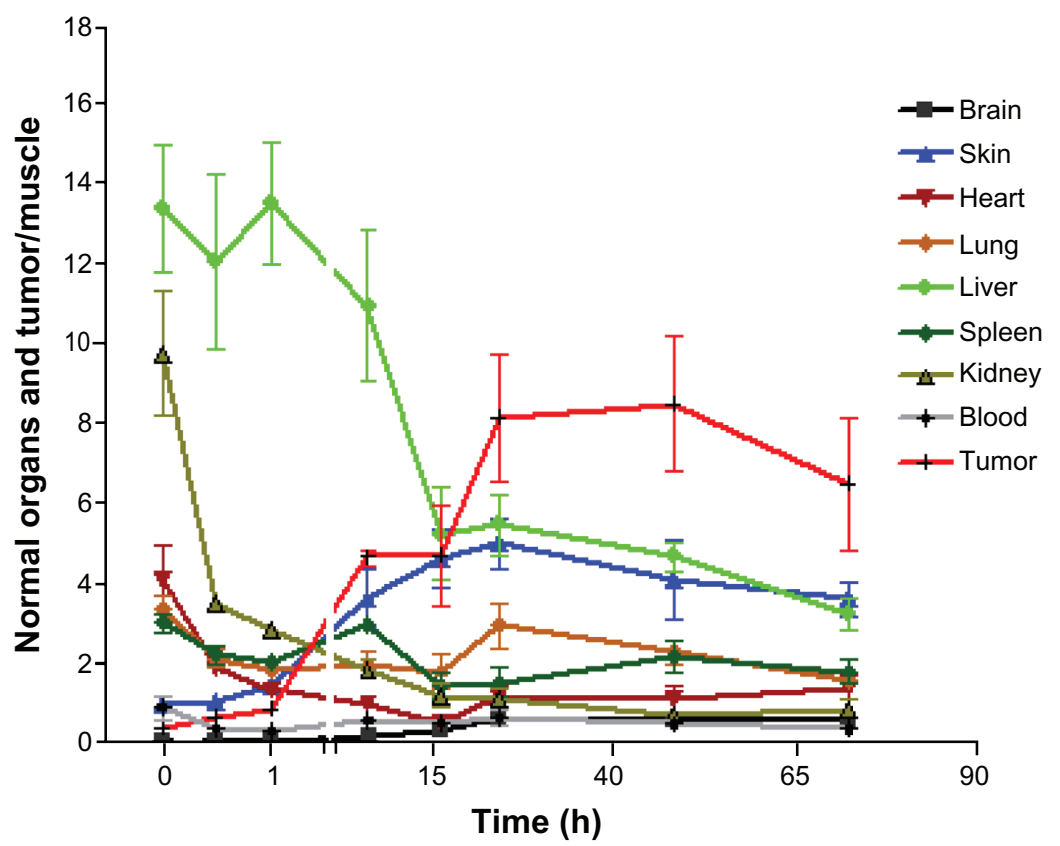

Figure 5A Normalized biodistribution of ${ }^{14} \mathrm{C}$-labeled paclitaxel loaded into nanomicelles in nude mice with SKOV-3 xenografts (organs/muscle).

The ratio of liver/muscle decreased dramatically within 16 hours post injection, even though it showed the highest ratio in the beginning. The ratio of skin/muscle increased up to around 5 -fold. The ratio of tumor/muscle kept increasing to a peak of around 8.5 -fold and showed much higher levels than those of organs/muscle after 16 hours. 


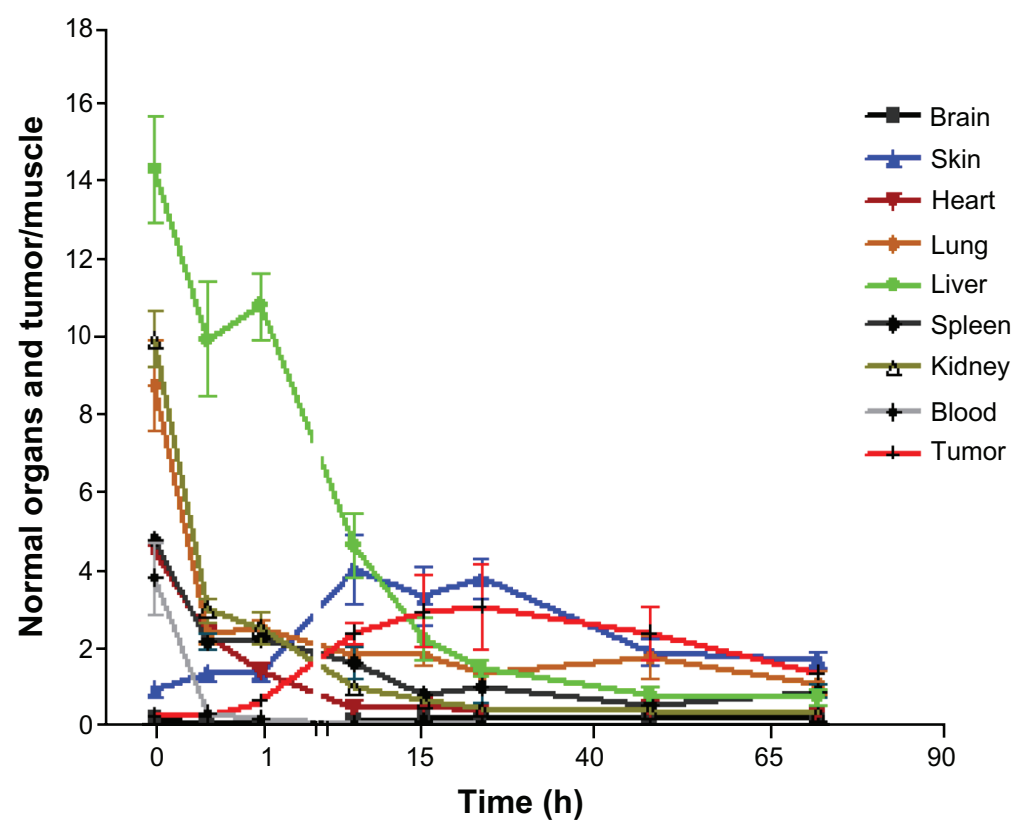

Figure 5B Normalized biodistribution of ${ }^{14} \mathrm{C}$-labeled paclitaxel in Taxol ${ }^{\circledast}$ in nude mice with SKOV-3 xenografts (organs/muscle).

The ratio of liver/muscle showed higher levels for 6 hours post injection, but decreased rapidly at later time points. The ratio of skin/muscle kept increasing to a plateau at 6 hours post injection, and decreased after 24 hours. This was even higher than the ratio of tumor/muscle most of the time, and was only around 3.5 -fold at its peak.

and crystals of paclitaxel were observed in the diluted Taxol solution (20× dilution by phosphate-buffered saline), whereas the telodendrimer nanomicelles loaded with paclitaxel at the same dilution remained very stable in size and solubility over months.

To demonstrate the relative trend of ${ }^{14} \mathrm{C}$-PTX uptake in different organs and tumor tissue, all values were normalized to muscle ${ }^{14} \mathrm{C}$ levels for each time point, except in blood (Figure 5). Most organ ratios displayed continuously decreasing radiocarbon ratios, except for skin, elimination from which was slow compared with muscle, which is consistent with the high lipophilicity of paclitaxel. For ${ }^{14} \mathrm{C}$-PTX-NM, the tumor/muscle ratio of radiocarbon content kept increasing and reached a plateau at approxi- mately 8.5 -fold 24-48 hours post injection. However, the normalized tumor/muscle ratio of ${ }^{14} \mathrm{C}$-PTX-Taxol showed an increase of only 3.5-fold at the highest point (Figure 5B), which is much lower than that when ${ }^{14} \mathrm{C}$-PTX was loaded in nanomicelles. Furthermore, the ratio of tumor/ muscle $\left({ }^{14} \mathrm{C}\right.$-PTX-Taxol) was even lower than that of the skin/muscle ratio at earlier time points at least 24 hours post injection (Figure 5B). These observations indicated that telodendrimer nanomicelles enhanced the uptake of paclitaxel by tumor tissue.

The whole blood pharmacokinetic profile of paclitaxel after administration of ${ }^{14} \mathrm{C}$-PTX-NM or ${ }^{14} \mathrm{C}$-PTX-Taxol at dose levels of $15 \mathrm{mg} / \mathrm{kg}$ was calculated using a onecompartment model (Figure 6). A $\mathrm{t}=0$ time point was used
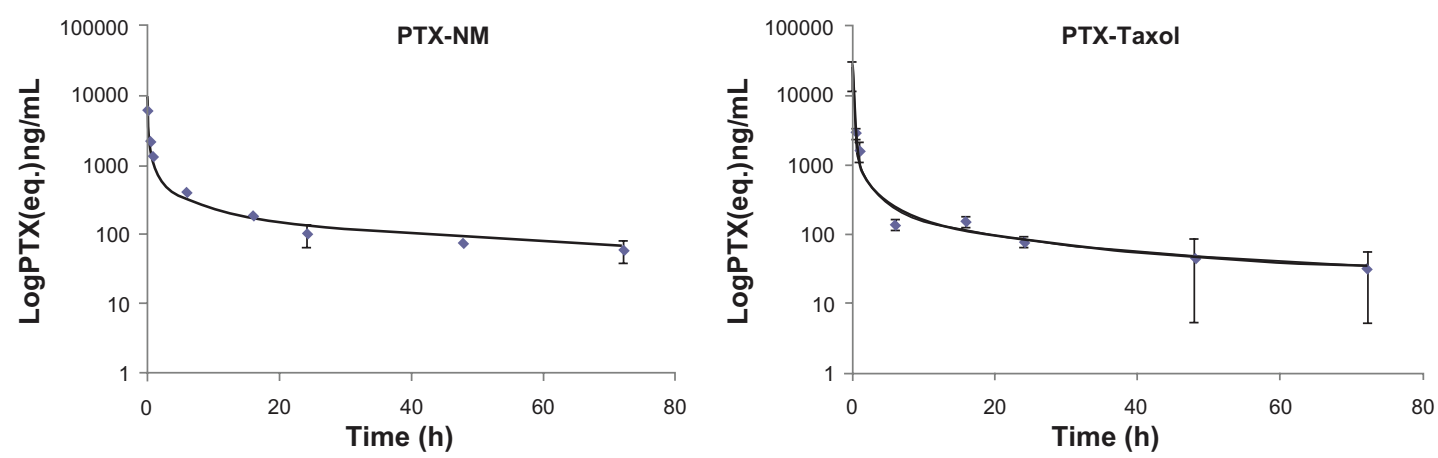

Figure 6 Pharmacokinetic data for paclitaxel formulated in nanomicelles and Taxol ${ }^{\circledR}$.

The terminal half-life ( $t_{1 / 2} 70.3$ hours) of paclitaxel loaded into $P E G^{5 k} C A_{8}$ micelles was significantly longer than that for Taxol ( $t_{1 / 2} 46.6$ hours, $P<0.05$ ). The area under the curve for PTX-NM was slightly higher, indicating modestly longer systemic exposure to the drug as a result of the nanomicelle formulation.

Abbreviations: PTX, paclitaxel; NM, nanomicelles. 
Table I Pharmacokinetic parameters for paclitaxel in whole blood

\begin{tabular}{|c|c|c|c|c|c|c|c|c|}
\hline \multirow{2}{*}{$\begin{array}{l}\text { Dose level } \\
(\mathrm{mg} / \mathrm{kg})\end{array}$} & \multirow[t]{2}{*}{ Formulation } & \multirow{2}{*}{$\begin{array}{l}C_{\max } \\
(\mathrm{ng} / \mathrm{mL})\end{array}$} & \multirow{2}{*}{$\begin{array}{l}\text { AUC } \\
(\mathrm{ng}-\mathrm{h} / \mathrm{mL})\end{array}$} & \multicolumn{3}{|c|}{ Elimination } & \multirow{2}{*}{$\begin{array}{l}V_{d} \\
(L / k g)\end{array}$} & \multirow{2}{*}{$\begin{array}{l}\mathrm{Cl} \\
(\mathrm{L} / \mathrm{h} / \mathrm{kg})\end{array}$} \\
\hline & & & & $\begin{array}{l}k \\
(1 / h)\end{array}$ & $\begin{array}{l}t_{1 / 2 \text { (beta) }} \\
\text { (h) }\end{array}$ & $\begin{array}{l}t_{1 / 2 \text { (gamma) }} \\
\text { (h) }\end{array}$ & & \\
\hline 15 & PTX-NM & 54730 & 21827 & 0.317 & 1.91 & 70.3 & 69758 & 687 \\
\hline 15 & PTX-Taxol & 20922 & 18306 & 0.153 & 3.15 & 46.6 & 54093 & 805 \\
\hline
\end{tabular}

Abbreviations: AUC, area under the concentration-time curve; $\mathrm{C}_{\max }$, peak plasma concentration; $\mathrm{V}_{\mathrm{d}}$, volume of distribution; Cl, clearance; PTX, paclitaxel; NM, nanomicelles.

to determine the maximum concentration of paclitaxel, which was $55 \mu \mathrm{g} / \mathrm{mL}$ for nanomicelles and $21 \mu \mathrm{g} / \mathrm{mL}$ for Taxol. The calculated initial absorption/distribution $\left(t_{1 / 2} \alpha\right)$ and terminal $\left(t_{1 / 2} \beta\right)$ half-lives were 1.9 hours and 70 hours, respectively, for nanomicelles, and 3.1 hours and 47 hours, respectively, for Taxol (Table 1). The area under the blood concentration-time curve for the nanomicelles was similar to that for Taxol (21.8 $\mu \mathrm{g}$-hours/mL and $18.3 \mu \mathrm{g}$-hours $/ \mathrm{mL}$, respectively). The blood half-lives for Taxol were similar to values reported for nude mice, except for the terminal half-life, which was previously reported to be 17 hours in plasma. ${ }^{17}$ The long terminal half-lives for both formulations in this report are consistent with paclitaxel uptake into blood cells, which is not accounted for in typical plasma or serum pharmacokinetic data. Although the initial whole blood absorption and distribution kinetics were faster for Taxol, the nanomicelle formulation persisted at higher concentrations at the later time points, possibly accounting, in part, for its improved preclinical efficacy. ${ }^{7,18-20}$

The longer half-life of PTX-NM compared with Taxol is consistent with slower hepatic elimination as a consequence of the $10-100 \mathrm{~nm}$ size of the nanomicelle nanocarrier. ${ }^{8}$ The slower blood terminal half-life observed for the nanomicelles is consistent with the slower hepatic filtration of the nanoparticle formulation, which has been previously reported for other nanoparticle-mediated drug delivery systems. ${ }^{21,22}$ The paclitaxel clearance of $805 \mathrm{~L} /$ hour $/ \mathrm{kg}$ for the Taxol formulation was reduced to $687 \mathrm{~L} / \mathrm{hour} / \mathrm{kg}$ for the PTX-NM formulation. Despite the fact that higher blood levels are reached when paclitaxel is given in the Taxol formulation, the tissue levels were essentially similar for both of the tested drug preparations.

It has to be noted that two different radiolabeling strategies were used in this research, in which the radio isotope ${ }^{125} \mathrm{I}$ was conjugated to telodendrimers for the SPECT imaging study and ${ }^{14} \mathrm{C}$-PTX was loaded inside nanomicelles for the pharmacokinetic experiments. There was a high radioactive signal found in the bloodstream during the first 24 hours in the microSPECT/CT images (Figures 1 and 3A). However, in the pharmacokinetic study, ${ }^{14} \mathrm{C}$-PTX-NM showed much less accumulation in the bloodstream than in the rest of the body, except for muscle, even within one minute post injection (Figure 4A). One explanation for this is that the current nanomicelle formulation is not stable in blood and that the nanomicelles disassemble partially and the loaded drug begins to release from the nanomicelles immediately after in vivo administration and diffusion into normal organs, resulting in a rapid decrease of ${ }^{14} \mathrm{C}$-PTX in the circulation. ${ }^{23,24}$ In contrast, the ${ }^{125} \mathrm{I}$-telodendrimers, even if coming from dissociated nanomicelles, still remain in the blood circulation for a longer time, contributing to a much higher radioactive signal in the blood during the 24 hours post injection.

We recently reported on the development of disulfide or boronate-catechol cross-linked nanomicelles ${ }^{23,24}$ that are much more stable than the non-crosslinked micelles reported here. The main advantage of crosslinked micelles is that premature drug release in the circulation can be minimized. Furthermore, disulfide reduction can occur inside the tumor cells with high glutathione levels, or on demand with exogenously administered N-acetyl cysteine, a US Food and Drug Administration approved reducing agent. Similarly, the bornate catechol crosslinkages can be reversed with the acidic environment at the tumor site and inside the endosomes of the tumor cells, or with exogenously administered mannitol, an approved diuretic. As a result, such crosslinked nanomicelles may be therapeutically more efficacious than non-crosslinked micelles. ${ }^{23,24}$ Work is underway in our laboratory using the techniques described in this study to determine the biodistribution and pharmacokinetics of such crosslinked nanomicelles.

\section{Conclusion}

The tumor-targeting properties of the telodendrimer $\mathrm{PEG}^{5 \mathrm{k}} \mathrm{CA}_{8}$ nanomicelle system have been previously demonstrated through near-infrared fluorescence optical imaging with improved preclinical outcomes compared with Taxol and Abraxane ${ }^{\circledR}$. MicroSPECT/CT imaging enabled by ${ }^{125}$ I-labeling of the nanomicelles further verified the highly efficient tumor targeting of the nanomicelles and provides a potential nanoplatform for cancer imaging and diagnosis. The biodistribution study of ${ }^{14} \mathrm{C}$-PTX indicates that sequestration 
of the drug in the $\mathrm{PEG}^{5 \mathrm{k}} \mathrm{CA}_{8}$ nanomicelle system markedly prolongs the paclitaxel circulation time in blood and improves the uptake of paclitaxel at the tumor site. The liver was the organ showing the highest uptake of paclitaxel at the beginning but the uptake had decreased rapidly to lower than tumor levels by 16 hours post injection. The results of radioimaging and biodistribution of the loaded drug support potential use of the $\mathrm{PEG}^{5 \mathrm{k}} \mathrm{CA}_{8} \mathrm{NM}$ system as a promising nanocarrier for imaging-guided drug delivery.

\section{Acknowledgments}

The authors are grateful for the financial support of NIH/ NCI R01CA140449 (JL), R01CA1 15483 (KSL), NIH/NCRR RR 1313461 and NIH/NCI 1R01CA155642 (PTH), Prostate Cancer Foundation (KSL), and the California Breast Cancer Research Program 14IB-0075 (PTH, TJ). The authors also thank the Center for Molecular and Genomic Imaging, UC Davis, for its imaging contributions, supported in part by a UC Davis Cancer Center support grant (P30CA093373, $\mathrm{NCI}$ ).

\section{Disclosures}

The authors report no conflicts of interest in this work. KL is the founding scientist of LamnoTherapeutics Inc, which plans to develop the nanotherapeutics described in the manuscript.

\section{References}

1. Lammers T, Kiessling F, Hennink WE, et al. Nanotheranostics and image-guided drug delivery: current concepts and future directions. Mol Pharm. 2010;7(6):1899-1912.

2. Mahmoudi M, Sant S, Wang B, et al. Superparamagnetic iron oxide nanoparticles (SPIONs): development, surface modification and applications in chemotherapy. Adv Drug Deliv Rev. 2011;63(1-2):24-46.

3. Petri-Fink A, Chastellain M, Juillerat-Jeanneret L, et al. Development of functionalized superparamagnetic iron oxide nanoparticles for interaction with human cancer cells. Biomaterials. 2005;26(15):2685-2694.

4. Jiao PF, Zhou HY, Chen LX, et al. Cancer-targeting multifunctionalized gold nanoparticles in imaging and therapy. Curr Med Chem. 2011;18(14): 2086-2102.

5. Kuijpers SA, Coimbra MJ, Storm G, et al. Liposomes targeting tumour stromal cells. Mol Membr Biol. 2010;27(7):328-340.

6. van Vlerken LE, Amiji MM. Multi-functional polymeric nanoparticles for tumour-targeted drug delivery. Expert Opin Drug Deliv. 2006;3(2): 205-216.

International Journal of Nanomedicine

\section{Publish your work in this journal}

The International Journal of Nanomedicine is an international, peerreviewed journal focusing on the application of nanotechnology in diagnostics, therapeutics, and drug delivery systems throughout the biomedical field. This journal is indexed on PubMed Central, MedLine, CAS, SciSearch ${ }^{\circledR}$, Current Contents ${ }^{\circledR} /$ Clinical Medicine,
7. Xiao K, Luo J, Fowler WL, et al. A self-assembling nanoparticle for paclitaxel delivery in ovarian cancer. Biomaterials. 2009;30(30): 6006-6016.

8. Luo J, Xiao K, Li Y, et al. Well-defined, size-tunable, multifunctional micelles for efficient paclitaxel delivery for cancer treatment. Bioconjug Chem. 2010;21(7):1216-1224.

9. Gurfinkel M, Ke S, Wen X, et al. Near-infrared fluorescence optical imaging and tomography. Dis Markers. 2003;19(2-3):107-121.

10. Mahmood U, Tung CH, Bogdanov A Jr, et al. Near-infrared optical imaging of protease activity for tumor detection. Radiology. 1999; 213(3):866-870.

11. Ke S, Wen X, Gurfinkel M, et al. Near-infrared optical imaging of epidermal growth factor receptor in breast cancer xenografts. Cancer Res. 2003;63(22):7870-7875.

12. Cherry SR. Multimodality in vivo imaging systems: twice the power or double the trouble? Annu Rev Biomed Eng. 2006;8:35-62.

13. Xiao K, Luo J, Li Y, et al. The passive targeting of polymeric micelles in various types and sizes of tumor models. Nanosci Nanotechnol Lett. 2010;2:79-85

14. Sparreboom A, van Tellingen O, Nooijen WJ, et al. Determination of paclitaxel and metabolites in mouse plasma, tissues, urine and faeces by semi-automated reversed-phase high-performance liquid chromatography. J Chromatogr. 1995;664(2):383-391.

15. Beijnen JH, Huizing MT, ten Bokkel Huinink WW, et al. Bioanalysis, pharmacokinetics, and pharmacodynamics of the novel anticancer drug paclitaxel (Taxol). Semin Oncol. 1994;21(5 Suppl 8):53-62.

16. Li C, Yu DF, Inoue T, et al. Synthesis, biodistribution and imaging properties of indium-111-DTPA-paclitaxel in mice bearing mammary tumors. J Nucl Med. 1997;38(7):1042-1047.

17. Sparreboom A, van Tellingen O, Nooijen WJ, et al. Nonlinear pharmacokinetics of paclitaxel in mice results from the pharmaceutical vehicle Cremophor EL. Cancer Res. 1996;56(9):2112-2115.

18. Sparreboom A, van Zuylen L, Brouwer E, et al. Cremophor EL-mediated alteration of paclitaxel distribution in human blood: clinical pharmacokinetic implications. Cancer Res. 1999;59(7):1454-1457.

19. Kumar GN, Walle UK, Bhalla KN, et al. Binding of Taxol to human plasma, albumin and alpha 1-acid glycoprotein. Res Commun Chem Pathol Pharmacol. 1993;80(3):337-344.

20. Wild MD, Walle UK, Walle T. Extensive and saturable accumulation of paclitaxel by the human platelet. Cancer Chemother Pharmacol. 1995;36(1):41-44.

21. van Vlerken LE, Duan Z, Little SR, et al. Biodistribution and pharmacokinetic analysis of Paclitaxel and ceramide administered in multifunctional polymer-blend nanoparticles in drug resistant breast cancer model. Mol Pharm. 2008;5(4):516-526.

22. Wang C, Wang Y, Wang Y, et al. Characterization, pharmacokinetics and disposition of novel nanoscale preparations of paclitaxel. Int $J$ Pharm. 2011;414(1-2):251-259.

23. LiY, Xiao K, Luo J, et al. Well-defined, reversible disulfide cross-linked micelles for on-demand paclitaxel delivery. Biomaterials. 2011;32(27): 6633-6645.

24. LiY, Xiao W, Xiao K, et al. Well-defined, reversible boronate crosslinked nanocarriers in response to acidic $\mathrm{pH}$ and diol concentrations. Angew Chem Int Ed Engl. January 17, 2012. [Epub ahead of print.] 